\title{
OTIMIZAÇÃO DA DOSAGEM DE AGLOMERANTE NO PROCESSO DE PRODUÇÃO DE PELOTAS DE MINÉRIO DE FERRO EM DISCO DE PELOTAMENTO PELO MODELO DE PREDIÇÃO DO RETORNO*
}

\author{
João Luis Moreira ${ }^{1}$ \\ Luis Alberto Sfalsin ${ }^{2}$ \\ Alexandre Juvenal Anacleto ${ }^{3}$ \\ Arthur Trindade Klein ${ }^{4}$ \\ Tiago Botti Castelo 5 \\ Gleidson Martins dos Santos ${ }^{6}$ \\ Priscilla Messias Pereira da Silva ${ }^{7}$ \\ Evandro Marchesi ${ }^{8}$
}

\section{Resumo}

Com o aumento da eficiência dos processos produtivos, faz-se cada vez mais necessária à utilização de técnicas e ferramentas que proporcionem a otimização de processos a fim de se extrair deles a máxima capacidade produtiva, com o mínimo de custo e variação. Neste contexto, observa-se que ações automatizadas, tomadas por sistemas que possuam inteligência pré programadas, são capazes de realizar este feito, inferindo nos processos por meio de ações otimizadas, na medida e hora certa, utilizando regras e ferramentas matemáticas adequadas. A eficácia destas técnicas já são comprovadas na Samarco. Este artigo está voltado para otimização da dosagem de aglomerante na planta 3 de pelotização da Samarco em Ubu com objetivo de reduzir a variabilidade, reduzir o retorno de pelotas cruas, aumentar a quantidade de pelotas na faixa entre 8 a $16 \mathrm{~mm}$ e, consequente, aumento da produtividade.

Palavras-chave: Eficiência; Otimização; Dosagem; Produtividade.

\section{OPTIMIZATION THE BINDER DOSAGE IN THE PROCESS OF IRON ORE PELLETS PRODUCTION IN PELLETIZING DISC BY RETURN PREDICTION MODEL}

\section{Abstract}

With the increased efficiency of production processes is made increasingly necessary the use of techniques and tools that provide process optimization in order to extract from them the maximum capacity with minimal cost and variation. In this context it is observed that automated actions taken by systems that have pre-programmed intelligence are able to accomplish this feat, inferring the processes through optimized shares to the extent and the right time, using rules and appropriate mathematical tools. The effectiveness of these techniques are proven in Samarco. This article attempts to optimize the binder dosage in the plant 3 of Samarco pelletizing in Ubu in order to reduce variability, reduce the return of raw pellets, increase the amount of pellets in the range of 8 to $16 \mathrm{~mm}$ and consequent increase in productivity.

Keywords: Efficiency; Optimization; Dosage; Productivity.

1 Eng. Metalúrgico e de Materiais, Laboratorista, Dep. Eng. de Processo, Samarco Mineração S/A, Anchieta, ES, Brasil.

2 Eng. de Produção, Engenheiro de Processo, Dep. Eng. de Processo, Samarco Mineração S/A, Anchieta, ES, Brasil.

3 Técnico Mecânico, Técnico de Processo, Dep. Eng. de Processo, Samarco Mineração S/A, Anchieta, ES, Brasil

4 Técnico Mecânico, Laboratorista, Dep. Eng. de Processo, Samarco Mineração S/A, Anchieta, ES, Brasil.

5 Técnico Metalúrgico, Laboratorista, Dep. Eng. de Processo, Samarco Mineração S/A, Anchieta, ES, Brasil.

6 Técnico Metalúrgico, Técnico de Processo, Dep. Eng. de Processo, Samarco Mineração S/A, Anchieta, ES, Brasil.

7 Eng. Metalúrgico e de Materiais, Eng. de Processo, Dep. Produção Usinas III e IV, Samarco Mineração S/A, Anchieta, ES, Brasil.

8 Eng. Químico, Técnico de Processo, Dep. Eng. de Processo, Samarco Mineração S/A, Anchieta, ES, Brasil. 


\section{INTRODUÇÃO}

Conceitualmente, a Pelotização consiste na mistura de finos de minério de ferro, com determinada teor umidade, acrescido de quantidades pré-definidas de aglomerante, óxidos básicos e energéticos. Os arranjos físicos e mecânicos dispostos pelos discos de pelotização implicam na ação da tensão superficial de capilaridade, promovendo a formação de um aglomerado esférico de tamanho entre 8,0 e $16,0 \mathrm{~mm}$, que posteriormente são submetidas a tratamento térmico, propiciando a resistência mecânica ao manuseio e propriedades metalúrgicas, quando submetidas à alimentação dos fornos de redução como carga metálica.

As condições operacionais do pelotamento em discos favorecem a aglomeração dos finos de minério de ferro. Contudo, é a umidade presente a grande responsável pela união das partículas, que pode ser pontencializada pela adição de aglomerante orgânico e ou mineral. $\mathrm{O}$ que difere os agentes aglomerantes é a incorporação de elementos químicos indesejados na pelota, tais como: $\mathrm{SiO} 2, \mathrm{Al} 2 \mathrm{O} 3$ e outros, provenientes dos minerais. Os orgânicos são nobres, pois não interferem na composição química da pelota, conferindo controle na taxa de crescimento e resistência (compressão e teste de queda) até processo de endurecimento.

Em virtude das propriedades do aglomerante não influenciar na química da pelota, sua utilização no processo é dada por controle convencional de difícil documentação e aprendizado.

Os sistemas especialistas utilizam como entrada de dados os conhecimentos adquiridos por pessoas especialistas das áreas operacionais e de engenharia. Outro ponto positivo é que os modelos de controle aplicam em suas regras desde ferramentas convencionais a algoritmos e modelos preditivos (dependendo do nível de complexidade e de informações inseridas no sistema)

O trabalho tem como objetivo apresentar os ganhos advindos da utilização do controle otimizante na dosagem de aglomerante na planta de pelotização da Samarco Mineração e sua capacidade de controle na redução da variabilidade do processo, maximizando a produção de pelotas cruas entre as faixas granulométrica de $8,0 \mathrm{~mm}$ à $16,0 \mathrm{~mm}$ e, consequentemente, aumento da produtividade dos fornos.

\section{MATERIAIS E MÉTODOS}

\subsection{Processo de Pelotização}

Segundo Meyer [1], a etapa de aglomeração trata-se de um processo dinâmico, influenciado por características intrínsecas do minério e insumos, além de condições dependentes da atuação do operador para obtenção da faixa granulométrica desejada. A busca do controle de todas estas variáveis de processo é uma tarefa complexa. A figura 1 mostra de modo resumido como o operador toma a decisão de dosagem de aglomerante para controle da taxa de crescimento da pelota antes da implementação deste projeto. 


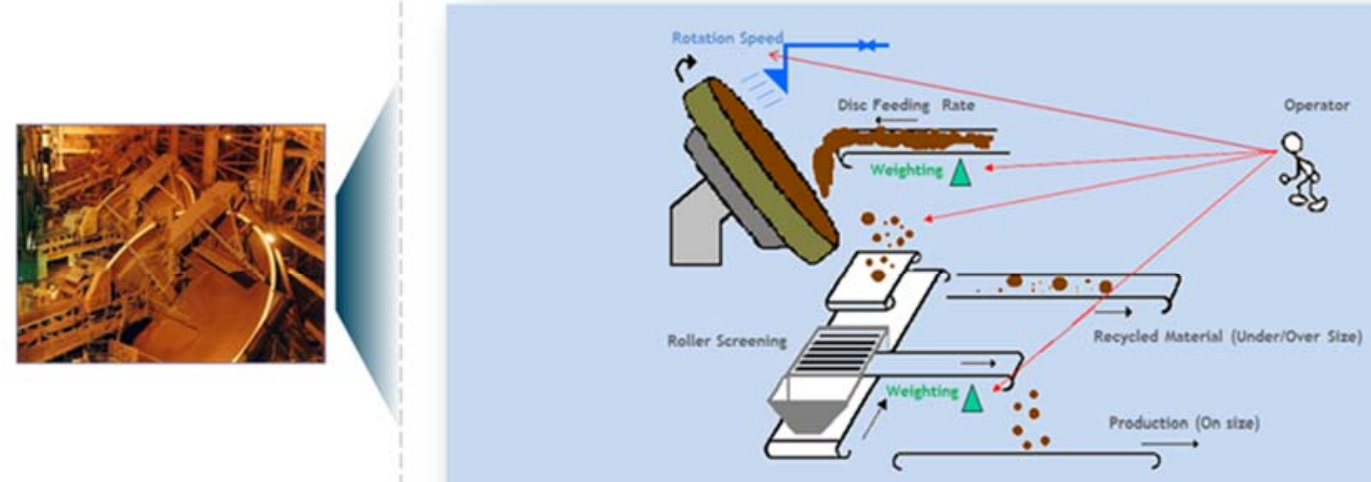

Figura 1 - Controle manual do processo de pelotamento

\subsection{Operação Manual de Dosagem}

A dosagem manual depende exclusivamente da atuação dos operadores do pelotamento na busca do tamanho desejado da pelota. O operador faz o pedido de dosagem e monitora, por observações visuais, a granulometria naquele dado momento. Além disso, servem de informações as análises de umidade do pellet feed, resistência ao número de queda, compressão de pelota crua, sendo estes resultados gerados a cada 2 horas pelo laboratório físico.

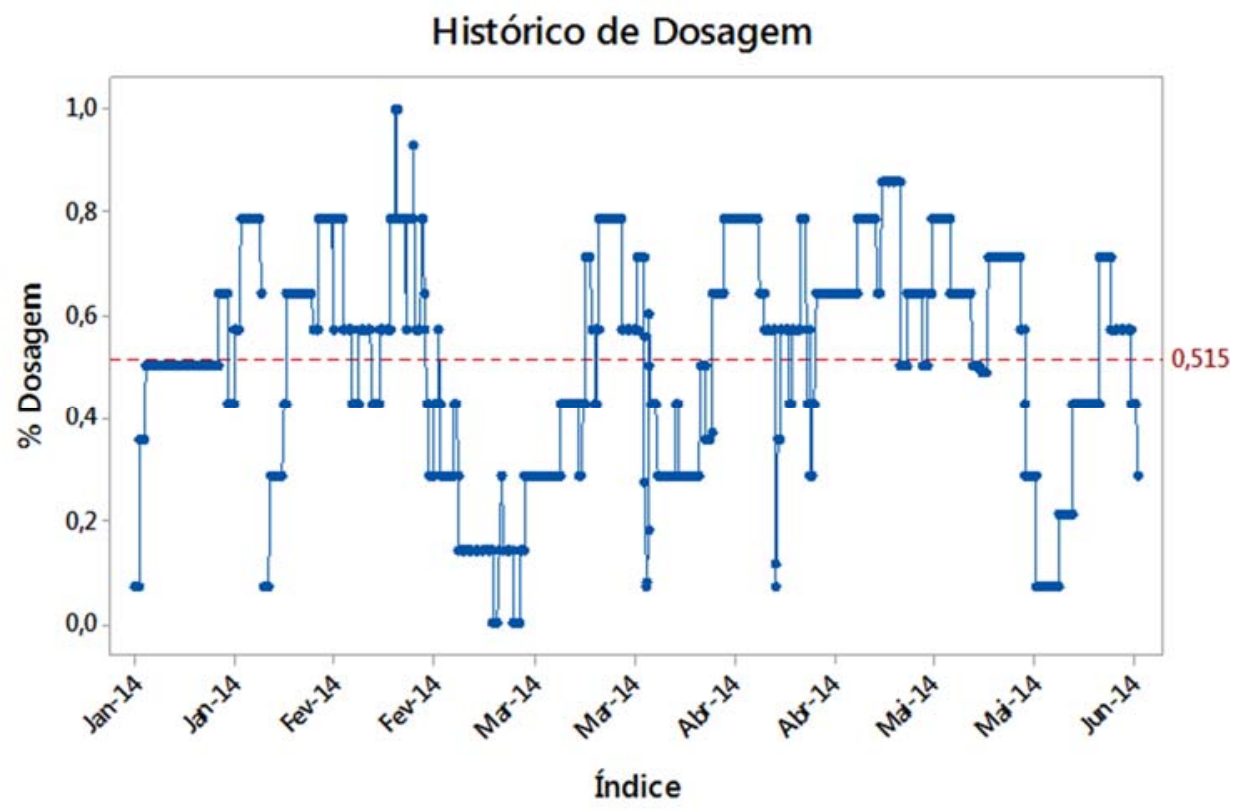

Figura 2 - Dosagem de aglomerante orgânico

Os dados históricos de dosagem do aglomerante demostram variação. Além disso, chama a atenção períodos longos estáticos (sem mudanças de dosagens). Isso se deve ao ato das atividades rotineiras exigirem tempo dos operadores para execução, além da falta de exatidão na determinação da melhor dosagem para um determinado momento do processo. 


\subsection{Operação Otimizada de Dosagem}

A aplicação do controle otimizado, busca qual a melhor dosagem de aglomerante para se obter um menor retorno de pelotas cruas e, consequentemente, maior alimentação da grelha, com resultados de qualidade da pelota queimada satisfatória, levando-se em consideração as variações de momento das propriedades da matéria prima.

\subsection{Fluxo Macro do processo}

Com intuíto de compreender melhor o fluxo, foi utilizado o SIPOC(supplier -input process - output - custumer), objetivando assim explicar o processo de maneira macro. O SIPOC traz as principais entradas, saídas do processo, assim como fornecedores e clientes de cada etapa.
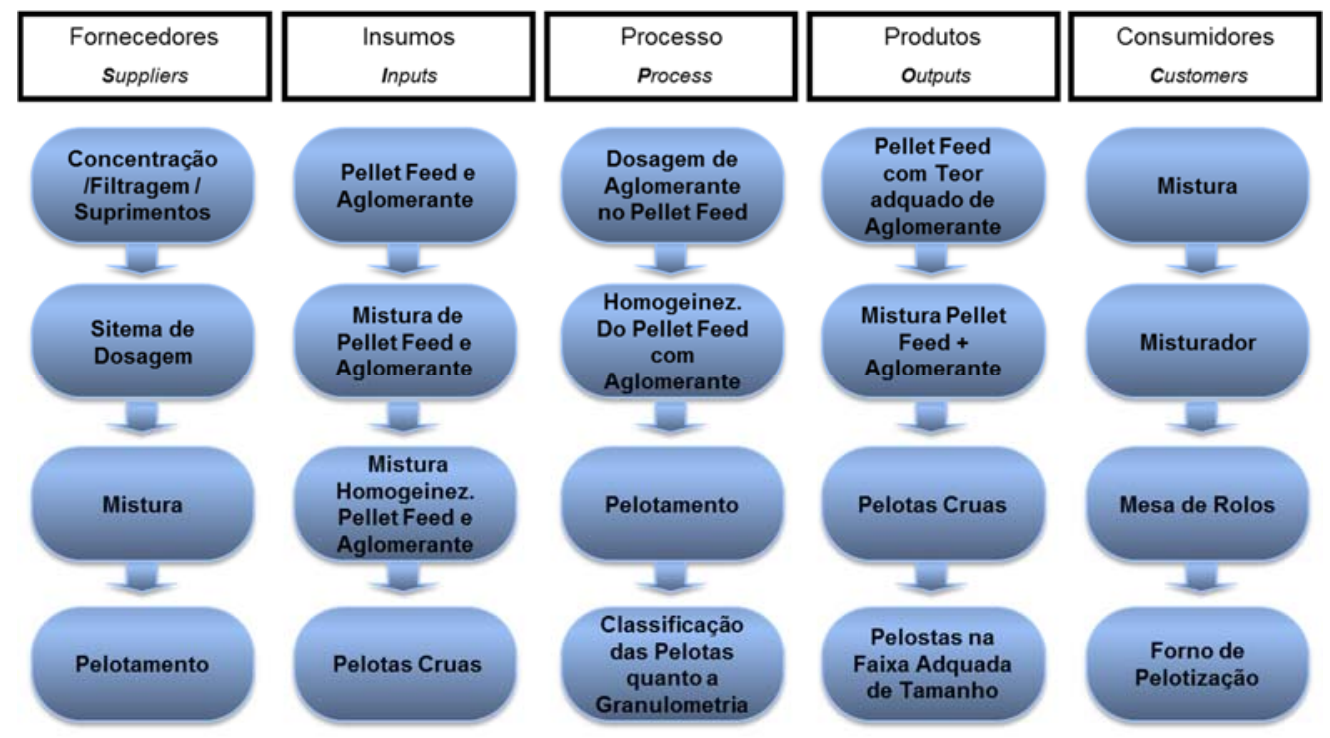

Figura 3 - Processo de forma Macro

O retorno do pelotamento é uma das etapas de processo que mais estão relacionadas às características críticas prioritárias. Com isso, esta variável foi priorizada na análise, com o objetivo principal de permitir que o sistema otimizante desenvolva o controle com base em manter esta variável resposta a menor possível. Isto faz da etapa de Pelotamento a etapa mais dependente da dosagem de aglomerante, porém todas as etapas se mostram igualmente importantes. 


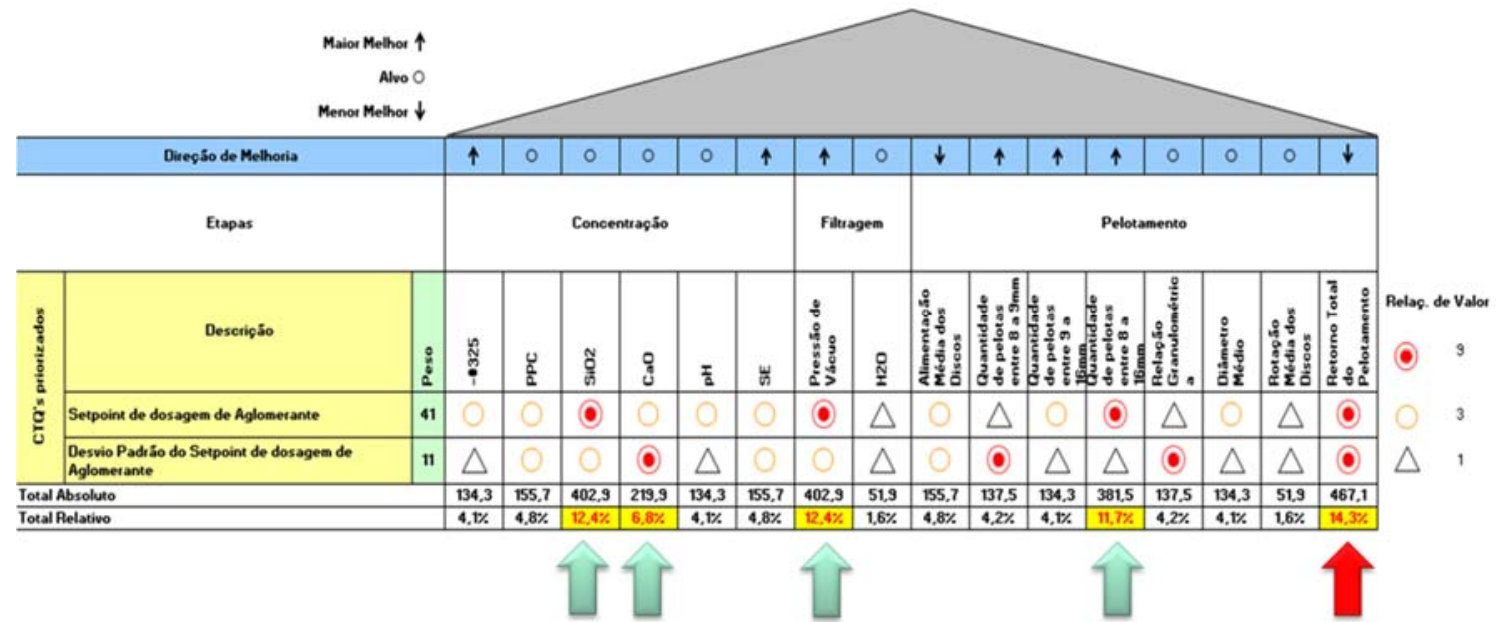

Figura 4 - Priorização das diretrizes estabelecidas

\subsection{Estratégia de controle}

O controle baseia-se em um modelo de previsão do retorno total, com o intuito de ajustar a dosagem de aglomerante de forma preventiva e antecipando-se às variações do retorno real e do tamanho da pelota crua.

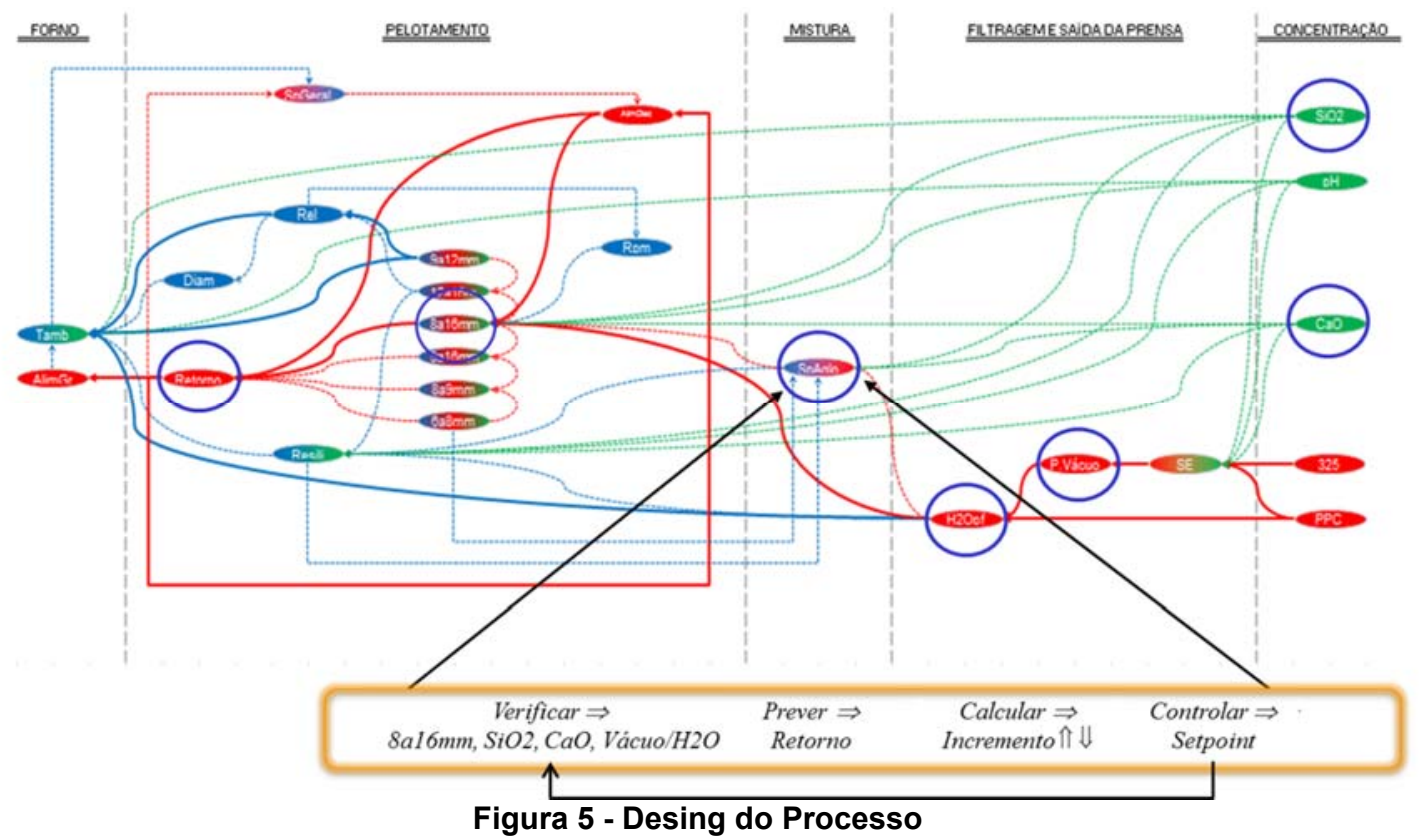

Cabe ressaltar que a estratégia de controle não atua nas características de composição química ou física do pellet feed. O objetivo é antecipar-se às variações do mesmo, visando uma melhora no desempenho dos processos seguintes (pelotamento e forno). A construção da estratégia de controle utilizou ferramentas estatísticas, regras fuzzy, regras crisp e cálculos matemáticos. As ferramentas foram trabalhadas em conjunto, fornecendo como solução os incrementos para se atingir as metas pré-estabelecidas. 


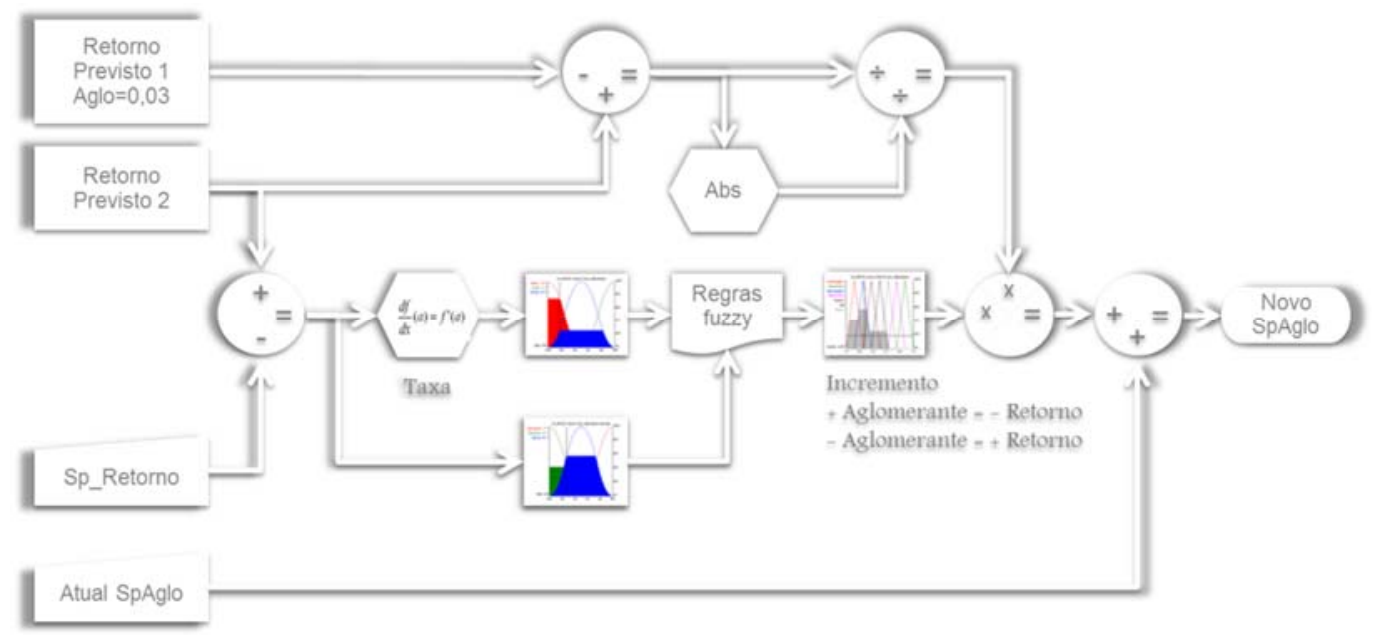

Figura 6 - Estratégia de Controle

Os incrementos deverão ser calculados periodicamente e enviados ao sistema de automação, via comunicação OPC, que por consequência manipulará o setpoint de dosagem de aglomerante. O modelo de regressão foi construído a partir das variáveis de maior correlação com o retorno do pelotamento.

\section{RESULTADOS E DISCUSSÃO}

Os sistemas otimizantes são maduros e confiáveis, uma vez que possuem como premissas os conhecimentos dos especialistas e análises estatísticas inseridas por meio de regras nos sistemas (para controle e otimização do processo). É fato que para obtenção de bons resultados de utilização dos sistemas, torna-se necessário a quebra de paradigmas, ou seja, mudança de cultura dos usuários. E a melhor maneira para isso é evidenciar os resultados com a implementação do controle.

Com o controle otimizante na operação da dosagem de aglomerante, novos patamares de dosagem foram explorados, propiciando redução do consumo de aglutinante, conforme figura 7 .

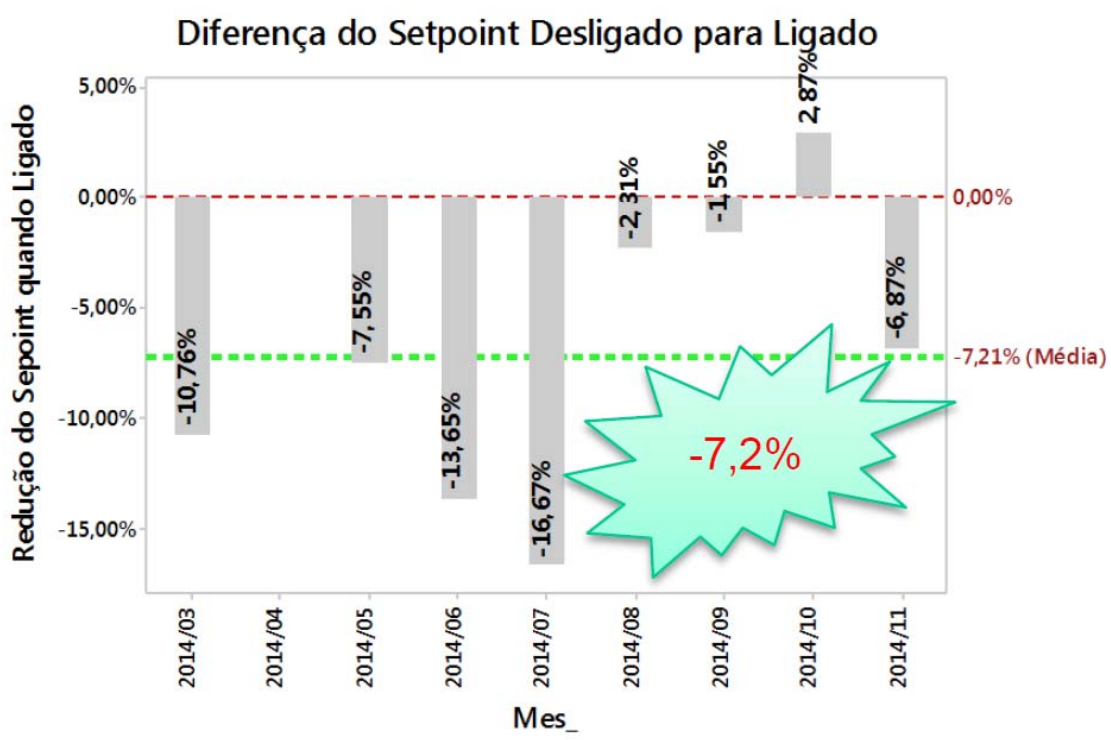

Figura 7 - Diferença de dosagens para controle Desligado e Ligado 
O sistema atendeu os principais propósitos de controle, otimizando a dosagem de aglomerante e reduzindo o percentual de pelotas fora de especificação em termos de taxa de crescimento. Importante frisar que, de maneira positiva, a otimização não implicou em alterações de qualidade da pelota queimada produzida.

\section{Boxplot de SetPoint-Agl; CCS -16,0+12; Tamboramento; Retorno Pelo}

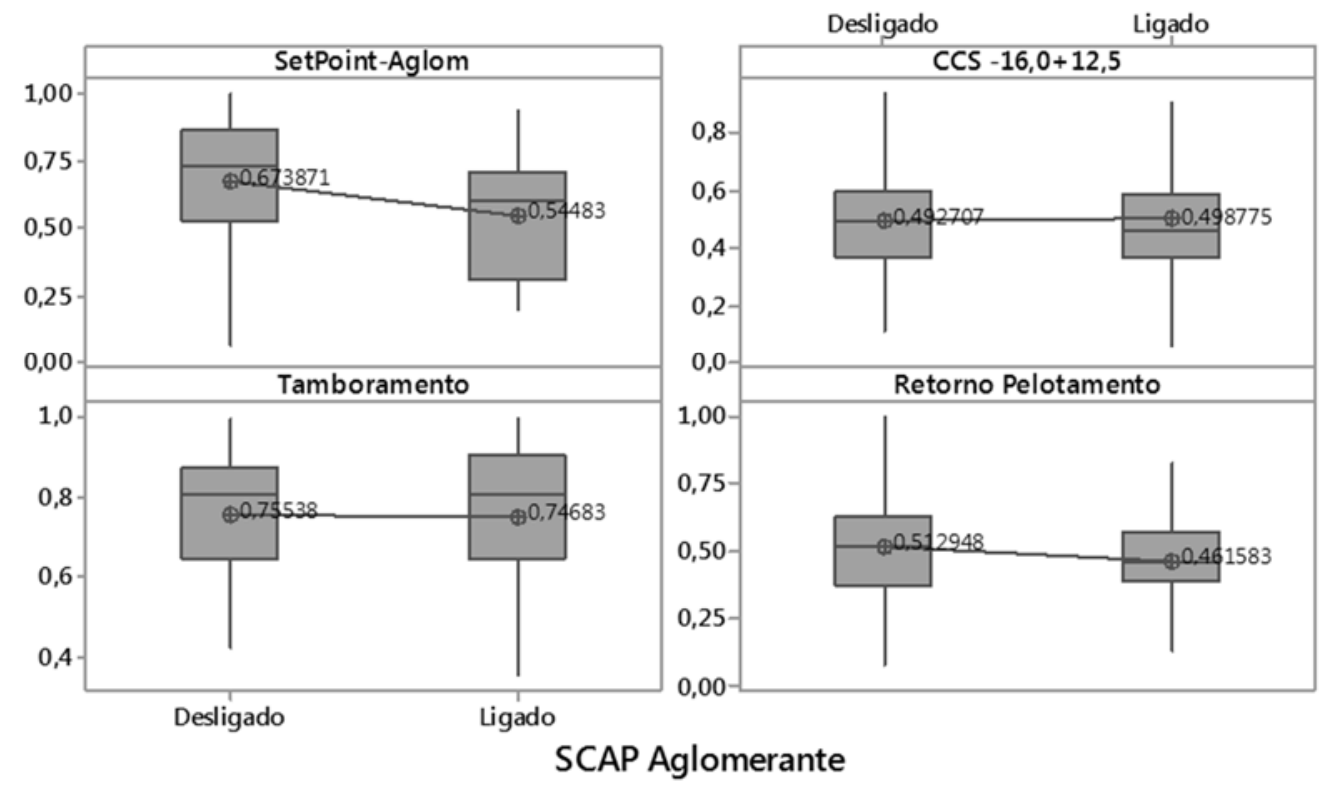

Figura 8 - Resultados do controle otimizando vs histórico

Confirma-se a diferença existente entre os períodos de dosagem (convencional e otzimada) pelo teste de Kruskal-wallis, que analisa se duas amostras independentes se originam de populações idênticas. Como o valor $p$ é menor do que nível alfa estatístico, considera-se uma mudanças nos valores de dosagem de aglomerante e o retorno total do pelotamento.

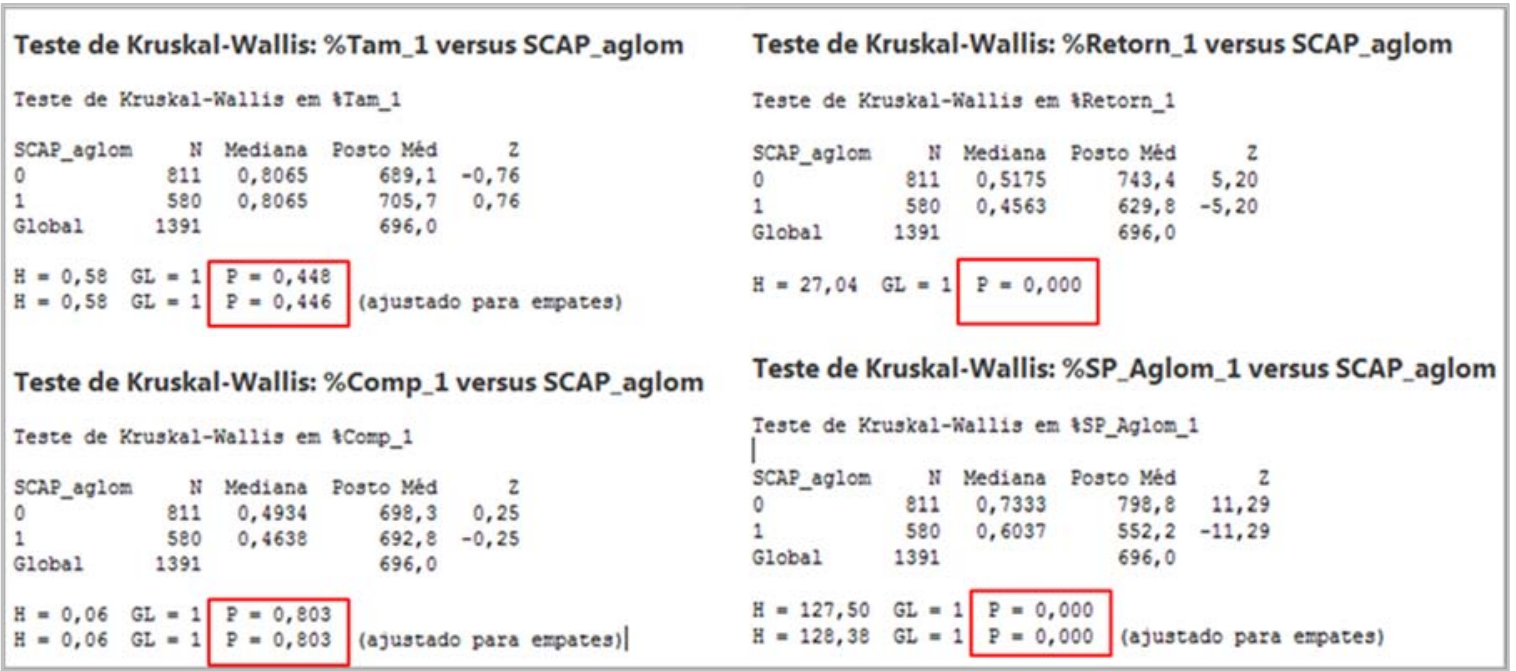

Figura 9 - Análise de mudança de patamar dos indicadores com controle otimizado 


\section{CONCLUSÃO}

O sistema de controle avançado de processo é uma alternativa madura de controle, precisa e robusta, desde que bem programado. Os resultados são notórios na redução da variabilidade proporcionada pela otimização da dosagem de aglomerante se comparados os dados antes e após a implementação. Como se previa, o sistema também foi capaz de reduzir custos de produção. Vale frisar que para obtenção desses resultados, é importante manter o sistema com alta disponibilidade e alta utilização.

\section{Agradecimentos}

Os autores agradecem a Samarco Mineração pelo suporte e incentivo.

\section{REFERÊNCIAS}

1 MEYER, Kurt - Pelletizing of Iron Ores. Germany: Springer - Verlag Berlin Heidelberg New York, 1980

2 SFALSIN, L.A.P. Seminário técnico para clientes da Samarco Mineração S/A, maio de 2014, Anchieta.

3 SFALSIN,L.A.P,MOREIRA,J.L,JORGE, A. Melhoria no desempenho do processo de produção de pelotas de minério de ferro em discos de pelotização pela utilização de sistemas otimizantes com lógica nebulosa. $44^{\circ}$ Seminário de Redução do Minério de Ferro e Matérias Primas, 15-17 de outubro de 2014, Minas Gerais. 